\title{
CARDINALITY OF DISCRETE SUBSETS OF A TOPOLOGICAL SPACE
}

\author{
David B, Gauld and M.K, Vamanamurthy
}

In this paper we prove two results relating the cardinalities of discrete subsets to those of dense subsets of a topological space. In particular, we show that

(1) closed discrete subsets of separable normal spaces are countable, and

(2) discrete subsets of separable regular spaces have cardinalities at most that of the continuum.

Theorem 4.1.15 of [1] shows that for any cardinal number $m$ and any metrizable space $X$ seven conditions are equivalent. Included in these seven are the following:

(a) every (closed) discrete subspace of $X$ has cardinality less than or equal to $m$;

(b) $X$ has a dense subset of cardinality less than or equal to $m$.

These conditions may be formulated in an arbitrary topological space and we consider how they are related in such a wider setting.

THEOREM 1. If the normal space $X$ has a dense subset of cardinality less than or equal to $m$ then every closed discrete subspace of $X$ has cardinality less than or equal to $m$.

Proof. Let $D$ be a dense subset of $X$ having cardinality less than

Received 14 August 1981. 
or equal to $m$, and let $C$ be a closed discrete subspace of $X$. Define a function $\varphi: P(C) \rightarrow P(D)$, where $P(Y)$ denotes the power set of a set $Y$, as follows. If $A \subset C$ then $A$ and $C-A$ are each closed in $X$ so by normality of $X$ there are disjoint open sets $U_{A}$ and $V_{A}$ with $A \subset U_{A}$ and $C-A \subset V_{A}$. Set $\varphi(A)=D \cap U_{A}$.

The function $\varphi$ is injective, for if $A, B \subset C$ with $A \neq B$, then either $A-B \neq \varnothing$ or $B-A \neq \emptyset$ : suppose the former. We have $\emptyset \neq A-B \subset U_{A} \cap V_{B} \subset U_{A}-c l U_{B}$. Since $U_{A}-c l U_{B}$ is an open set, it meets $D$. Thus

$$
\emptyset \neq D \cap\left(U_{A}-c I U_{B}\right) \subset \varphi(A)-\varphi(B) .
$$

Hence $\varphi(A) \neq \varphi(B)$, so $\varphi$ is injective.

Injectivity of $\varphi$ immediately implies the result.

Taking the particular case where the cardinal number $m$ is the first infinite cardinal, we obtain the following corollary of Theorem 1 .

COROLLARY 1. Closed discrete subsets of separable normal spaces are countable.

COROLLARY 2. Sorgenfrey's square $S$ is not normal.

Proof. The points having rational coordinates form a countable dense subset of $S$, so $S$ is separable. However, the (uncountable) subset consisting of all points on any given line of slope $-I$ is closed and discrete. Hence by Corollary $1, S$ is not normal.

Since Sorgenfrey's square is completely regular, we cannot replace normality in Theorem 1 by complete regularity. However we do obtain a related result if we assume only regularity.

THEOREM 2. If the regular space $X$ has a dense subset of cardinality less than or equal to $m$ then every discrete subspace of $X$ has cardinality less than or equal to $\exp m$.

Proof. Let $D$ be a dense subset of $X$ having cardinality less than or equal to $m$ and let $C$ be a discrete subspace of $X$. Define an infection $\varphi: C \rightarrow P(D)$ as follows. If $x \in C$, then there is an open subset $W_{x}$ of $X$ with $C \cap W_{x}=\{x\}$. By regularity of $X$, there is an 
open neighbourhood $U_{x}$ of $x$ with cl $U_{x} \sim W_{x}$. Set $\varphi(x)=D \cap U_{x}$.

To verify injectivity of $\varphi$, suppose $x, y \in C$ with $x \neq y$. Then $x \in U_{x}$ but $x \leqslant$ cl $U_{y}$, so the non-empty open set $U_{x}-$ cl $U_{y}$ meets $D$. But $D \cap\left(U_{x}-c I U_{y}\right) \subset \varphi(x)-\varphi(y)$. Thus $\varphi(x) \neq \varphi(y)$.

Injectivity of $\varphi$ leads to the result.

COROLLARY 3. Discrete subsets of separable regular spaces have cardinality at most that of the continum.

Since every topological group is regular, Theorem 2 and Corollary 3 are applicable here.

Theorem 1.5.3 of [1] tells us that if the Hausdorff space $X$ has a dense subset of cardinality less than or equal to $m$ then the cardinality of $X$ itself is at most exp exp $m$. However, for an arbitrary topological space there appears to be no relationship between the cardinalities of (closed) discrete subspaces and dense subsets. For example, let $X$ be a set of at least three elements and let $p \in X$. Topologise $X$ by $\{U \subset X \mid p \in U\} U\{\emptyset\}$. Then $\{p\}$ is dense in $X$ but $X-\{p\}$ is a closed discrete subspace of arbitrarily large cardinality.

\section{Reference}

[1] Ryszard Engelking, General topology (Monografie Matematyczne, 60. PWN - Polish Scientific Publishers, Warszawa, 1977).

Department of Mathematics, University of Auckland, Private Bag, Auckland, New Zealand. 